Revista de Ciencias Sociales - Número 70 (2017) - Páginas 13-25

El Ingreso Básico Universal como nuevo Derecho Social

\title{
EL INGRESO BÁSICO UNIVERSAL COMO NUEVO DERECHO SOCIAL
}

THE BASIC INCOME: A NEW SOCIAL RIGHT

\author{
ALEJANDRA ZÚÑIGA FAJURI* \\ Universidad de Valparaíso \\ alejandra.zuniga@uv.cl
}

\section{Resumen}

El trabajo desarrolla la propuesta del Ingreso Básico Universal que seduce hoy en Estados Unidos y Europa como posible alivio a los problemas del mercado de trabajo, para responder a las demandas del feminismo y para el cuidado del medioambiente. El Ingreso Básico Universal es una propuesta moralmente fundada en el principio de igualdad y libertad que busca garantizar un ingreso básico que permita a las personas alcanzar sus aspiraciones y planes de vida sin tener que, para ello, soportar relaciones humanas abusivas.

Palabras clave

Ingreso básico, igualdad, libertad.

* Doctora en Derecho por la Universidad Autónoma de Madrid. Centro de Investigaciones de Filosofía del Derecho y Derecho Penal (CIFDE), Universidad de Valparaíso, Errázuriz 2120, Valparaíso, Chile, alejandra.zuniga@uv.cl Artículo recibido el 24 de mayo de 2017 y aceptado el 6 de julio de 2017.

Revista de Ciencias Sociales - Número 70 (2017) - Universidad de Valparáíso - ISSN 0716-7725-Valparáiso, Chile 


\begin{abstract}
The paper develops the proposal of the Universal Basic Income that seduces today in the United States and Europe as possible relief to the problems of the labor market, the demands of feminism and the environment concerns. The Universal Basic Income is a proposal that's morally founded on equality and freedom principles and seeks to guarantee a basic income that allows people to achieve their aspirations and plan of life, without having to endure abusive human relationships.
\end{abstract}

\title{
Keywords
}

Basic income, equality, freedom.

\section{INTRODUCCIÓN}

"De cada cual (voluntariamente) según sus capacidades, a cada cual (incondicionalmente) según sus necesidades" (P. Van Parijs). Esta es la máxima en la que descansa el derecho al Ingreso básico universal, desarrollado hoy por quienes consideran necesario abrir un debate intelectualmente honesto y moralmente serio sobre los problemas sociales más importantes de la población, entre los que cabe destacar la tarea pendiente de garantizar la justa igualdad de oportunidades para todos que, en su esencia, implica asegurar los bienes básicos susceptibles de — en palabras de Amartya Sen_- "convertir recursos en libertades"1. Es lo que Rawls proponía como bases sociales mínimas necesarias para garantizar recursos básicos o "bienes primarios" que permitan alcanzar los planes de vida que cada cual ha delineado para sí.

Este trabajo desarrollará la propuesta del Ingreso Básico Universal que seduce hoy en Estados Unidos y Europa como posible alivio a los problemas del mercado de trabajo, como lo son los bajos salarios - en el caso de los EE.UU._- y el enorme desempleo — en el caso de Europa. Con todo, el principal argumento en favor del Ingreso Básico Universal

1. SEN, Amartya: "Equality of What?". En: Liberty, Equality and Law. Selected Tanner Lectures on Moral Philosophy. University of Utah Press - Cambridge University Press, 1987.

Facultad de Derecho y Ciencias Sociales - Universidad de Valparaíso - Chile 
es de libertad: Garantizar a todos un ingreso básico que nos permitiría estar en mejor posición para perseguir nuestras aspiraciones y planes de vida personales, lejos de trabajos extenuantes, mal pagados y de relaciones humanas abusivas. El Ingreso Básico Universal significa más libertad para todos y por ello es una idea moralmente atractiva que vale la pena estudiar.

\section{El Ingreso Básico Universal es tendencia}

El derecho a un Ingreso Básico Universal se ha extendido por todo el mundo. En Oakland, California (EEUU) el stanup "Y Combinator" anunció recientemente (en junio) que será sede del primer experimento de renta básica universal en los Estados Unidos². Alrededor de 100 familias recibirán un ingreso mínimo de entre $\$ 1.000$ y $\$ 2.000$ dólares cada mes para estudiar cómo dicho ingreso básico afecta a su felicidad, bienestar y la forma en que gastan su tiempo. Oakland fue elegido por su diversidad social y económica y por el hecho de que cuenta con gran concentración de riqueza y desigualdad. Se seleccionaron al azar a los participantes sin distinguir entre empleados y cesantes ${ }^{3}$.

En Ontario, Canadá, se ha comunicado (también recientemente) que pronto podría estar enviando un cheque mensual a sus residentes para poner en marcha el experimento del "ingreso básico". Aunque los oficiales en la provincia canadiense no han publicado aún detalles específicos del proyecto, el Ministerio de Finanzas divulgó un informe

2. Lo cierto es que el Fondo Permanente de Alaska el primer caso de IBU en el mundo. En 1976, al finalizarse la construcción del oleoducto de la Bahía de Prudhoe, Jay Hammond, gobernador de Alaska de 1974 a 1982, propuso la constitución de un fondo que tuviera como objetivo beneficiar a los habitantes mediante el depósito de una parte de los ingresos provenientes de la extracción de petróleo. Ese mismo año se aprobó la constitución del Alaska Permanent Fund, proveyéndose el capital inicial a partir de las licencias para la explotación de crudo. Desde 1982 el fondo paga a todo quien haya vivido en Alaska por a lo menos un año, un dividendo anual de aprox. 2 mil dólares, a modo de IBU.

3. Resulta interesante considerar que la empresa de Silicon Valley propuso la idea como una forma de contrarrestar los posibles problemas derivados de la tecnología de sustitución de puestos de trabajo existentes.

Revista de Ciencias Sociales - Número 70 (2017) - Universidad de Valparáíso - ISSN 0716-7725-Valparaíso, Chile 
que confirma la intención del gobierno de poner en marcha el experimento. "A medida que crece la economía de Ontario, el gobierno mantiene su compromiso de no dejar a nadie atrás. El mantenimiento de una red efectiva de seguridad social es una parte de los esfuerzos del gobierno para reducir la pobreza y garantizar la inclusión en las comunidades y la economía".

A su turno, en Gran Bretaña, el Think tank de la Royal Society para el Fomento de las Artes y el Comercio ha propuesto un sistema de ingreso básico para las personas en edad de trabajar, confiados en que no generará un desincentivo al trabajo. Se sugiere proporcionar un ingreso de $3.692 £$ anuales para todos los ciudadanos de entre 25 y 65 años, o $£ 308$ por mes $^{5}$. Estudios e iniciativas similares se están desarrollando en Finlandia, Nueva Zelanda, Alemania, Holanda, Francia y Suiza. En África, la organización GiveDirectly — creada para transferir pagos en efectivo a personas pobres - ha iniciado el pago durante 10 años a 6000 personas en Kenia y Uganda dentro de la figura de la "renta básica".

En una economía como la chilena, con gran precariedad en el empleo y un alto porcentaje de población con peligro de caer en la pobreza (Contreras, 2004) ${ }^{7}$ esta puede ser una herramienta crucial

4. "El piloto también podría probar si una renta básica proporciona una manera más eficiente de entregar las ayudas, fortaleciendo el vínculo con la fuerza de trabajo e incentivando el ahorro en áreas tales como la salud y la vivienda. El gobierno trabajará con las comunidades, investigadores y otras partes interesadas en 2016 para determinar la mejor manera de implementar un piloto de Renta Básica”. Declaración del presupuesto de Ontario del año 2015.

5. John McDonnell, the shadow chancellor, said the Labour party would not rule out a basic income for all members of society during a talk at the London School of Economics. "It's an idea we want to look at. Child benefit was a form of basic income so it's not something that I would rule out". Disponible en http:// www.independent.co.uk/news/world/americas/ontario-to-pilot-a-universal-basicincome-experiment-a6916571.htm

6. Disponible en https://www.givedirectly.org/basic-income.

7. CONTRERAS GUAJARDO, D., NEILSON, C., HERMANN ANGUITA, J. y COOPER, R. "The Dynamics of Poverty in Chile”, 2008, Disponible en http://www.repositorio.uchile.cl/handle/2250/127633

Facultad de Derecho y Ciencias Sociales - Universidad de Valparaíso - Chile 
destinada a recuperar la estabilidad social democrática, siempre en peligro en una sociedad tan desigual. "Argentina, Chile, Costa Rica, Uruguay y hasta Brasil están cerca de poder instaurar un ingreso mínimo universal o ciudadano sin condicionamientos y dar así el salto del sistema básico de protección social al de bienestar" (Martín Hopenhayn. Ex Director de la División de Desarrollo Social de la Comisión Económica para América Latina y el Caribe- CEPAL) ${ }^{8}$.

\section{Una propuesta liberal igualitaria}

El Ingreso Básico Universal es una propuesta que se mantiene fiel a las dos intuiciones básicas del liberalismo igualitario: igual preocupación por los intereses de cada cual (esta es la dimensión igualitaria) e igual respeto hacia las distintas concepciones de la vida buena (esta es la dimensión liberal), sin sesgos “perfeccionistas". Ser "liberal”, en este sentido filosófico, no significa ser pro-mercado o pro-capitalismo. Significa, en cambio, sostener que una sociedad justa no debe estar fundamentada en una concepción previa de lo que debe ser una vida buena de modo que, por ejemplo, tanto la heterosexualidad como la homosexualidad merecerán el mismo respeto. Una concepción liberal supone que es posible definir qué es una sociedad justa sin apoyarse en una concepción del "bien" particular.

El liberal igualitario (o de izquierda) estima como injusto que los miembros de una misma sociedad dispongan de medios desiguales para desarrollar su concepción de la vida buena. Lo justo es, entonces, que todos inicien su vida con igualdad de recursos e igualdad de oportunidades, de modo que una sociedad "liberal igualitaria" deberá justificarse si se aparta de este principio. Ahora, garantizar que todos inicien su vida con iguales recursos no asegurará la igualdad de resultados pues, en primer lugar, debemos considerar la responsabilidad personal que puede motivar que algunos dispongan posteriormente de más recursos porque han hecho elecciones distintas: han trabajado más, han ahorrado más, han pasado más tiempo formándose, han corrido

8. Véase en http://www.ipsnoticias.net/2010/12/se-asoma-la-renta-basicauniversal-por-america-latina/

Revista de Ciencias Sociales - Número 70 (2017) - Universidad de Valparáíso - ISSN 0716-7725-Valparáiso, Chile 
más riesgos, etc. "La justicia, entonces, consiste en repartir las posibilidades de forma equitativa, no los resultados. Lo que cada cual haga con sus posibilidades es su responsabilidad individual. He aquí, pues, una primera consideración que permite apartarse de la igualdad sin caer en la injusticia"”.

La segunda consideración que debe tenerse presente es la eficiencia. Hay situaciones en las que un cierto nivel de desigualdad contribuye tanto a la eficiencia que incluso las "víctimas" de tal desigualdad se ven beneficiadas de ella (los "peor situados" tendrían aún menos si la situación fuera menos desigual). Este es el famoso "principio de diferencia” de John Rawls ${ }^{10}$. Es el mercado el que decide el valor que dará al trabajo de cada cual. Y cuando digo mercado, digo demanda, cuando digo demanda, digo preferencia de las personas, de todos nosotros. La idea central del Ingreso Básico Universal admite que el mercado es una institución que ya no nos abandonará. "El mecanismo de los precios es un dispositivo prodigioso que logra condensar en una métrica única informaciones relativas a millones de preferencias de naturaleza e intensidad muy diversas, por un lado, y millones de recursos de naturaleza muy desigual, por el otro" ${ }^{11}$.

Con todo, el mercado es totalmente incapaz de generar de forma espontánea una distribución equitativa de los recursos sociales, por lo que resulta fundamental lograr una buena articulación entre mercado y democracia. Van Parijs y Van der Veen llaman al Ingreso Básico Universal "la vía capitalista al comunismo" pues consistiría en "capitalizar" el dinamismo del capitalismo del cual hablaba Karl Marx para incrementar gradualmente la proporción del producto social a redistribuir en relación a las necesidades de las personas ${ }^{12}$. Se busca,

9. VAN PARIJS, Philippe: Renta básica, capacidades y necesidades. Disponible en www.sinpermiso.info publicado el 21 julio 2013.

10. RAWLS, John: A Theory of Justice, Oxford University Press, 1971.

11. VAN PARIJS, Philippe, ob.cit.

12. VAN PARIJS, Philippe, y VAN DER VEEN, Robert J., "Una vía capitalista al comunismo”. En: Zona abierta, No 46-47, 1988. Págs. 19-46.

Facultad de Derecho y Ciencias Sociales - Universidad de Valparaíso - Chile 
luego, reducir gradualmente el número de personas empleadas en actividades alienantes o en trabajos muy mal pagados y ayudarlos a encontrar un trabajo que tenga sentido para ellos.

\section{4. ¿̇qué es el Ingreso Básico Universal?}

El Ingreso Básico Universal es un ingreso pagado por el gobierno, de manera uniforme y en intervalos regulares, a todo miembro adulto de la sociedad. El ingreso es fijo y se paga por igual con independencia de si la persona es rica o pobre, vive sola o con otros, trabaja o está desempleada ${ }^{13}$. En la versión de Van Parijs el ingreso se entrega tanto a nacionales como a residentes permanentes. Se llama "básico" al ingreso pues es una base material con la que se puede contar con seguridad para subsistir. Cualquier otro ingreso derivado del trabajo o de los ahorros se puede sumar a él sin alterarlo. La idea es que el monto -que puede discutirse - alcance para mantener una "vida decente" (Van Parijs considera que, en los países desarrollados, es posible entregar un ingreso que esté por sobre el "nivel de subsistencia") ${ }^{14}$.

Finalmente, vale la pena aquí destacar la obra de Joseph Charlier "Solution of the social problem" donde argumenta que cada ciudadano tiene derecho a un "dividendo territorial" en razón de la propiedad compartida e igual sobre el territorio de la nación ${ }^{15}$. Este es el argumento que sustenta la famosa obra de Van Parijs, "Real Freedom for All: What (if Anything) Can Justify Capitalism?" ${ }^{16}$. Entonces ¿Qué no es un Ingreso Básico Universal?

Resulta importante no confundir la propuesta del Ingreso Básico Universal con otras propuestas que, aunque similares, poseen carac-

13. VAN PARIJS, Philippe, ROGERS, Joel y COHEN, Joshua (eds.): "What's Wrong With a Free Lunch?”, Beacon Press, Boston, 2001.

14. Ídem.

15. Sobre la historia del IBU, desde Charles Fourier a Milton Friedman, véase pp. 4-5 de la obra citada.

16. VAN PARIJS, Philippe: Real Freedom for All, What (if anything) can justify capitalism. Oxford: Clarendon Press, 1995.

Revista de Ciencias Sociales - Número 70 (2017) - Universidad de Valparáíso - ISSN 0716-7725-Valparaíso, Chile 
terísticas que las hacen menos atractivas. En efecto, como se señaló, las características del IBU son: individual, universal e incondicional. Estas tres características lo hacen totalmente diferente de las políticas basadas en la evaluación de los medios económicos de que disponen las personas, como la propuesta de Velasco del impuesto negativo al trabajo o el mejor denominado "crédito fiscal por ingreso" (el Earned Income Tax Credit (EITC) promovido por el ex presidente de los EEUU, Bill Clinton, importado del Reino Unido de Tony Blair (Working Families Tax Credit) y promovido por la Francia de Jospin con el nombre de Prime pour l'Emploi).

En términos simples, el Impuesto negativo al ingreso (INI) es un beneficio solo para quienes trabajan y tienen ingresos bajos a moderados. Es un crédito fiscal reembolsables por lo que si el crédito es superior al importe de los impuestos adeudados, el exceso se devuelve al contribuyente. En principio se puede obtener lo mismo con el INI que con el Ingreso Básico Universal, y es posible incluso que el INI sea más barato de administrar pues evita el vaivén que resulta de pagar el Ingreso Básico Universal a quienes tienen ingresos suficientes, para luego pedir su devolución vía impuestos.

Aun así, el Ingreso Básico Universal tiene tres ventajas importantes sobre el INI. Primero, el INI solo será funcional si es capaz de garantizar - con pagos adelantados - que las personas no se mueran de hambre mientras esperan que su formulario de impuestos sea examinado al término de cada año fiscal. Pero sabemos que los programas de seguridad social suelen tener que lidiar con errores y confusiones que podrían privar a las personas que lo necesitan de ese pago por adelantado ${ }^{17}$. Segundo, aunque el INI puede operar en términos individuales, normalmente opera y es propuesto a nivel de "familiar". Por ello, aunque el INI y el Ingreso Básico Universal puedan ser iguales en términos netos, la distribución "intra-hogar" puede distar mucho de ser justa. Especialmente en el caso de las mujeres.

Finalmente, en tercer lugar, el Ingreso Básico Universal lidia mucho mejor que el INI con la llamada "trampa del desempleo", destacada por los trabajadores sociales pero usualmente ignorada por

17. Ídem.

Facultad de Derecho y Ciencias Sociales - Universidad de Valparaíso - Chile 
los economistas. Aunque lo racional para las personas sea buscar un buen empleo o cambiarse a uno mejor, la "incertidumbre" que se vive en el tiempo intermedio, cuando no se tienen ahorros, desmotiva a la gente a mejorar su situación. El IBU, en cambio, permite a las personas contar con ingresos mínimos suficientes para poder buscar alternativas laborales mejores, sin miedo a pasar hambre.

A su turno, el Ingreso Básico Universal también ha sido llamado "Ingreso ciudadano" (Stakeholder Society) que, en la propuesta de Bruce Ackerman y Anne Alstott — creada inicialmente por Thomas Paine - también está en desventaja con el Ingreso Básico Universal pues la propuesta consiste en entregar a todo ciudadano que alcance los 21 años una sola cantidad de dinero para que comience su vida adulta (aprox. 80 mil dólares). El problema evidente de esta propuesta surge cuando recordamos que, a lo largo de la vida, existirán miles de oportunidades para que las personas menos educadas o precavidas gasten todo su dinero torpemente, quedando nuevamente en la pobreza.

\section{Las razones morales y sociales que demandan el reconocimiento de este derecho}

i) Justicia: El principal argumento a favor del Ingreso Básico Universal es la justicia social. Ella requiere que la sociedad garantice a todos los individuos los recursos necesarios para la libertad. Esta concepción de "liberalismo real" busca una sociedad de personas formalmente libres (titulares de derechos básicos de libertad) y la garantía del valor real de esos derechos de libertad. El "Valor de la libertad" - worth of liberty, en palabras de Rawls — depende de los recursos que posea una persona para poder hacer efectiva esa libertad ${ }^{18}$. (Siguen esta línea Elizabeth Anderson, Amartya Sen, Gerald Cohen, Will Kymlicka, entre muchos otros).

ii) Crecimiento versus trabajo: En principio, la introducción del Ingreso Básico Universal —en un contexto de desarrollo del capitalismo cognitivo, el capitalismo cada vez más basado en el conocimientoson razones de orden ético que refuerzan la exigencia de combinar

18. Ídem.

Revista de Ciencias Sociales - Número 70 (2017) - Universidad de Valparaíso - ISSN 0716-7725-Valparaíso, Chile 
trabajos de baja remuneración con los beneficios que emanan de una forma universal de renta de existencia. El argumento no es que el capitalismo cognitivo tienda a producir una demanda de trabajo cada vez menor (aunque esa es la tesis de autores como Jaron Lanier, en su famoso ¿Quién controla el futuro?) sino más bien que tiende a distribuir el poder derivado de las ganancias de un modo cada vez más asimétrico, llegando incluso a reducir los salarios del grueso de quienes se hallan por debajo de un nivel de vida decente (Como ha ocurrido en los últimos 100 años en los países desarrollados, según ha demostrado Thomas Piketty en "El capital en el siglo XXI") ${ }^{19}$.

Luego, desde un punto de vista más bien político, el Ingreso Básico Universal aparece como la única medida capaz de resolver el dilema entre un modelo europeo de pobreza limitada y alto desempleo con el modelo estadounidense de bajo desempleo pero extendidos focos de pobreza. En el primer caso, durante los últimos 30 años, se ha instalado en la mayoría de los países europeo-occidentales un masivo desempleo que no han podido resolver con el recurso clásico de la teoría económica: el aumento del crecimiento económico. Ello pues, por una parte, la tecnología (capitalismo cognitivo) está eliminando rápidamente una gran cantidad de empleos y, por otra parte, un acelerado crecimiento económico — que solo serviría para no aumentar más el desempleo, difícilmente para reducirlo_ - no parece la vía más adecuada desde el punto de vista ecológico.

La alternativa, en la línea del modelo estadounidense, es reducir drásticamente los salarios. Así los efectos negativos de la tecnología disminuirían, sacrificándose menos trabajos. Claro que el problema de esto es que implica imponer un inaceptable nivel de pobreza a gran parte de la población.

iii) Feminismo y medioambiente: Durante el gobierno de Nixon en los Estados Unidos se efectuaron experimentos cuidadosamente seguidos de formas de impuesto negativo en distintos lugares. El primer efecto de estos experimentos fue una reducción no enorme, pero sí estadísticamente significativa, de la oferta de trabajo de los

19. PIKETTY, Thomas: El capital en el siglo XXI, Bogotá: Fondo de Cultura Económica, 2013.

Facultad de Derecho y Ciencias Sociales - Universidad de Valparaíso - Chile 
"secondary earners", esto es, de los miembros del hogar que aportan el segundo sueldo - mayoritariamente mujeres-. En segundo lugar, los índices de divorcio habían aumentado. ¿Qué refleja este fenómeno? Por un lado, que ciertas mujeres utilizaron la posibilidad de escapar de su doble jornada de trabajo, por el otro lado, que un cierto número de mujeres se dijeron: "estoy harta de este tipo, ahora que tengo un poco de autonomía financiera, me largo" ${ }^{20}$. Por ello, autoras feministas como Nancy Fraser, de Nueva York, o Anne Alstott, de Yale, ven en la renta básica un importante instrumento de emancipación ${ }^{21}$.

Finalmente, el Ingreso Básico Universal responde a otras disfunciones graves del mercado relacionadas con los precios, que son incapaces de dar cuenta de lo que los economistas llaman externalidades negativas, como la contaminación y la conservación de los recursos naturales no renovables para las generaciones futuras.

\section{CONCLUSIÓN}

El Ingreso Básico Universal trata de lo que Thomas Paine llamó "el derecho a la existencia" o Charles Fourier, un "mínimo de subsistencia abundante”. Robespierre declaraba “¿Cuál es el primer fin de la sociedad? Mantener los derechos imprescriptibles de las personas. ¿Cuál es el primero de esos derechos? El de existir. La primera ley social es, pues, la de asegurar a todos los miembros los medios de existencia. Todas las demás se subordinan a ésta; la propiedad no ha sido instituida ni garantizada sino para cimentar aquella ley; es para vivir que se tienen las propiedades"22.

Difícilmente sea posible responsabilizar a los niños de la pobreza en la que viven. Como muestra Jon Roemer, tampoco es fácil respon-

20. VAN PARIJS, Philippe: Renta básica, capacidades y necesidades. Disponible en www.sinpermiso.info publicado el 21 julio 2013.

21. ELGARTE, Julieta: "Basic Income and the Gendered Division of Labour". En: Basic Income Studies, vol. 3, No 3, Article 4, 2008.

22. DOMÉNECH, Antoni, El eclipse de la fraternidad, una revisión republicana de la tradición socialista, Ed. Crítica, 2004. Pág. 82. Disponible en Polis [En línea]. Publicado el 29 octubre 2012.

Revista de Ciencias Sociales - Número 70 (2017) - Universidad de Valparáíso - ISSN 0716-7725-Valparáiso, Chile 
sabilizar a un adulto de las condiciones precarias que su pobreza inicial de alguna manera ha condicionado. La pobreza no sólo es consecuencia del azar natural, sino que también (y especialmente) del azar social, de las reglas sociales de distribución y adjudicación de bienes que hemos instaurado desde hace siglos sobre la base de principios de justicia (piénsese en las reglas de adjudicación de la propiedad privada de Locke) que hoy no resultan sostenibles (Rawls).

Si no podemos moralmente justificar como justo (conforme a las teorías de la justicia) el actual estado de cosas, entonces debemos avanzar incluso más allá de los mecanismos redistributivos establecidos hasta el momento. Por ello, la garantía de un Ingreso Básico Universal que permita liberar a las personas (por el sólo hecho de ser personas y miembros de una misma comunidad) del estado de necesidad y miedo

provocado por la pobreza, resulta moralmente coercitivo como derecho humanos básico reconocible en Chile.

\section{BIBLIOGRAFÍA}

CONTRERAS GUAJARDO, D., NEILSON, C., HERMANN ANGUITA, J. y COOPER, R. (2008-05). The Dynamics of Poverty in Chile. Disponible en http://www.repositorio.uchile.cl/handle/ 2250/127633

DOMÉNECH, Antoni. El eclipse de la fraternidad, una revisión republicana de la tradición socialista, Ed. Crítica, 2004, 473, p. 82.

ELGARTE, Julieta. "Basic Income and the Gendered Division of Labour”. Basic Income Studies. Vol. 3 [2008], No 3, Article 4.

PIKETTY, Thomas. El capital en el siglo XXI, Fondo de cultura económica, 2013.

RAWLS, John. A Theory of Justice, Oxford University Press. 1971.

SEN, Amartya (1987) "Equality of What?" Liberty, Equality and Law. Selected Tanner Lectures on Moral Philosophy. University of Utah Press - Cambridge University Press.

VAN PARIJS y VAN DER VEEN. "Una vía capitalista al comunismo". Zona abierta, No 46-47, 1988, pp: 19-46.

VAN PARIJS, Philippe. Real Freedom for All, What (if anything) can justify capitalism: Clarendon Press, Oxford, 1995.

Facultad de Derecho y Ciencias Sociales - Universidad de Valparaíso - Chile 
VAN PARIJS. What's Wrong With a Free Lunch? Philippe van Parijs, Joel Rogers (Editor), Joshua Cohen (Editor). Beacon Press, Boston, 2001.

VAN PARIJS: Renta básica, capacidades y necesidades. www.sinpermiso. info 21 julio 2013. 